\title{
Coulisses
}

Revue de théâtre

\section{Macbeth de William Shakespeare au théâtre du peuple de Bussang}

Rédaction

\section{(2) OpenEdition}

12 Journals

Édition électronique

URL : http://journals.openedition.org/coulisses/1663

DOI : $10.4000 /$ coulisses. 1663

ISSN : 2546-9460

Éditeur

Presses universitaires de Franche-Comté

Édition imprimée

Date de publication : 1 février 1991

Pagination : 72-74

ISSN : 1150-594X

\section{Référence électronique}

Rédaction, «Macbeth de William Shakespeare au théâtre du peuple de Bussang », Coulisses [En ligne], 3 | Hiver 1991, mis en ligne le 04 juillet 2017, consulté le 22 octobre 2019. URL : http:// journals.openedition.org/coulisses/1663; DOI : 10.4000/coulisses.1663

Ce document a été généré automatiquement le 22 octobre 2019

Coulisses 


\title{
Macbeth de William Shakespeare au théâtre du peuple de Bussang
}

\author{
Rédaction
}

\section{NOTE DE L'ÉDITEUR}

Les représentations ont été données les 28,29 juillet, 4, 5, 10, 11, 12, 15, 18, 19, 24, 25, 26 août 1990 à 15 heures. Théâtre du Peuple, 88540 Bussang.

1 Un bel après-midi d'été. Il fait très beau, très chaud. Le pré transformé en parking est couvert de voitures. Le bâtiment est étonnant. Tout en bois, il n'a rien d'un théâtre. Il rappelle une ferme ou une grange vosgienne, en beaucoup plus grand, revue par un architecte fantaisiste. Le public fait la queue tranquillement pour prendre ses billets à un prix modique, puis déambule ou s'asseoit paisiblement sur la prairie. L'atmosphère est à la fête, calme et joyeuse. La trompe appelle à pénétrer dans l'arche de bois.

\section{Le théâtre du peuple de Bussang}

Le désir me vint donc de créer un théâtre qui fût accessible à tous, au peuple entier, sans exclusion de caste ni de fortune, et qui pût intéresser tous ceux, d'esprits même très divers, qu'il réunirait sur ses gradins.

(Maurice Pottecher ${ }^{1}$ )

2 En 1895, le poète Maurice Pottecher, rêvant de libérer le théâtre des préjugés et modes imposés aux écrivains et artistes par le monde restreint des scènes parisiennes, crée à Bussang, dans les Vosges, le Théâtre du Peuple. Cette expérience, la première du genre en province, connaît un vif succès, dès la première représentation du Diable marchand de goutte (1895), auprès d'un très vaste public, de tous milieux, toutes classes, toutes confessions.

3 Figuration, confection des décors, interprétation... tout le village participe à l'élaboration des spectacles, avec, pour unique motivation, le désir de réjouissance 
commune. Nulle recherche de profit, l'art demeure le maître mot, gravé sur le fronton de la scène : «Par l'art... pour l'humanité ». Maurice Pottecher réalise ainsi le rêve de Michelet : « Nourrissez le peuple du peuple!»

Drames, comédies et poèmes lyriques, de Poil de Carotte à La Passion de Jeanne-d'Arc, de Shakespeare à Molière, se succèdent sur la scène du Théâtre du Peuple, qui accueille des artistes tels que Marguerite Vannson, Louis Jouvet ou Robert Lamoureux...

En 1945, reconstruit pour la seconde fois, le théâtre voit sa direction confiée à PierreRichard Wilm (spectacles) et Germaine Kiener (organisation et administration), et sa seconde résurrection est marquée par l'émouvante représentation de Liberté.

Cet enthousiasme n'est en rien altéré par la mort de Maurice Pottecher en 1960, dont les créations, au nombre d'une trentaine, sont régulièrement reprises, mais celles-ci ne tardent pas à s'avérer dépassées, et 1973 marque le retour à Shakespeare, insufflé par Tibor Egervari.

\section{Macbeth}

7 Macbeth créée en 1902 à Bussang est reprise dans la traduction de Maurice Pottecher. «Je ne connaissais pas cette version, souligne Pierre Étienne Heymann, le metteur en scène, elle est écrite en vers rimés, dans une langue rocailleuse, refusant tout romantisme et approchant au plus près le rythme de la poésie shakespearienne. » Avec une troupe de comédiens composée de professionnels: Bernard Waver, Macbeth, Margot Lefevre, Lady Macbeth, Mathias Beyler, Macduff et des stagiaires du TNS, Pierre Étienne Heymann a restitué à cette adaptation les scènes les plus sanglantes, assassinat de Lady Macduff, comme les plus triviales, le portier de l'Enfer, jugées trop crues à l'époque.

8 Pas de grandes machineries, le lieu y suffit... Pierre Étienne Heymann peut affirmer son ambition de renouer avec les origines du théâtre shakespearien :

J'ai été bouleversé, le bois, l'architecture, j'ai eu l'impression de me trouver chez Shakespeare. Le fond de scène a été ouvert, la forêt était là, prête à entrer dans le théâtre... Tout est dit dans le texte, à condition de sortir ce « tout » du texte ; aussi, tout sera rendu concret au premier degré, à travers des solutions simples, et, d'ailleurs, pas toujours très faciles à trouver. Lorsque le texte parle de spectre, on verra un spectre, on verra des sorcières apparaître et la forêt se mettre en mouvement... La pièce est bâtie sur des moments de grande tension dramatique, suivis de sciènes comiques. Ici, à Bussang, les acteurs sont tout près du public, nous allons donc jouer à fond, en allant au bout des divers styles qui se télescopent...

9 Judicieuse exploitation du célèbre cadre de Bussang, manipulation du plateau, rétréci ou agrandi, au fil de cette dramaturgie, qui oscille entre réel et imaginaire, grande tour de bois en mouvement, portes rétractables, costumes de cuir aux couleurs fortes, chevaux, ânes, animaux, faisant irruption au sein des spectateurs, effets spéciaux et artifices confèrent à la pièce une intensité tout à la fois magique et lugubre, merveilleuse et tragique, sous la direction de Simone Burn, réalisatrice de la scénographie au son de madrigaux des $\mathrm{xVI}^{\mathrm{e}}$ et $\mathrm{xVII}^{\mathrm{e}}$ siècles, dirigés par Boris de Vinogradov. L'ascension terrifiante et chaotique des deux criminels légendaires atteint son apothéose dans le dernier acte, lorsque la forêt entre véritablement sur scène et avance sur les spectateurs grâce à l'ouverture du fond de scène. 

cette parole s'accomplira. Il le deviendra, mais au prix des pires trahisons, des meurtres les plus abominables, pris dans l'engrenage du crime, qui ne peut le mener qu'à sa propre perte, au terme d'un parcours hérissé d'aventures fantastiques et de combats féroces...

11

Ainsi, la tragédie, débute par l'apparition des sorcières annonciatrices du destin. Etonnantes! Alors que le tract publicitaire propose une reproduction de gravure du XIX siècle, conforme à la tradition, celles que nous voyons sont jeunes, fraîches et accortes, de jaune et rouge vêtues, plus proches $d u$ «fou » du roi que du diable. On a du mal à croire à leurs maléfices. La malédiction ne serait peut-être pas là où on la pense, extérieure à soi. Ces jeunes femmes jouent à prédire... et le jeu est pris au sérieux par un général victorieux qui se prend au jeu de l'ambition, poussé par une épouse lasse de n'avoir aucun rôle de premier plan, espèce de Madame Bovary, sans ancrage dans la réalité de son temps, au contraire de Lady Macduff, riche de ses enfants et de l'avenir qu'ils lui assurent. Macbeth aime son épouse, il lui dédie ses trahisons. Mais leurs assassinats communs loin de fortifier leur passion ne fait que les éloigner l'un de l'autre. Le vainqueur éclatant, passionnément épris de sa femme, se transforme en déchet humain au fur et à mesure qu'il lui sacrifie son honneur. La passion amoureuse, que l'ambition devrait embraser, ne suffit pas à faire vivre Macbeth. Lady Macbeth, quant à elle, se révèle incapable de réaliser impunément son rêve de grande figure de l'histoire. Elle paie de sa raison cette ambition qu'elle ne peut assumer. L'immense baldaquin, est le signe récurrent de ce couple qui se défait faute de trouver les valeurs communes qui lui permettraient de continuer à vivre ensemble. À une mise en scène au premier degré correspond l'histoire de ces époux qui se détruisent faute d'une énergie et d'un cynisme à la hauteur de leurs crimes.

On y perd la méditation de Shakespeare sur le sens de l'histoire, de l'existence, du rôle de l'homme dans le chaos originel. Par contraste, la mise en scène de Langhorf à Chaillot, trouve sa véritable force iconoclaste, de rappel à la vocation du théatre montreur de vérité.

Tout en appréciant la beauté des images qui leur étaient proposées, les spectateurs ne sy sont pas trompés. Certains, ont trouvé «le spectacle trop intellectuel " pour eux, "trop éloigné de leurs préoccupations et de leur expression "; d'autres pas assez « audacieux ». En cherchant dans ce lieu envoûtant autre chose qu'une belle illustration d'un monument de la culture occidentale, une leçon plus profonde que celle de la dégradation d'un couple, fût-il machiavélique, ces spectateurs exigeants retrouvaient le vœu de Maurice Pottecher :

Ce théâtre sera un éveilleur de conscience. Chacun des spectateurs viendra y chercher un plaisir et en emporter un sujet de réflexion sur sa destinée ou sur l'avenir de sa race. 


\section{NOTES}

1. Maurice Pottecher, l'aventure du théâtre populaire, un parcours proposé par Catherine Foki, MarieJosé Pottecher Onderet, Édition Serpenoise. 